\title{
D’Amico criteria
}

National Cancer Institute ( $\mathrm{NCl})$

\section{Source}

National Cancer Institute (NCI). D'Amico criteria.

A set of clinical criteria that is used to stratify prostate cancers into three risk categories to estimate cancer growth and spread. The criteria include Gleason score, prostatespecific antigen level, and clinical stage at diagnosis. 